\title{
RESEARCH ON THE MAKER TEACHER MOBILE TRAINING PLATFORM BASED ON BIG DATA ANALYSIS
}

\author{
Zhenghong Jiang* \\ Chongqing Vocational College of Transportation, Chongqing, China - thomasyangly@126.com
}

KEY WORDS: Maker Teachers; Big Data Analysis Technology; Professional Development of Teachers; Mobile Training Platform

\begin{abstract}
:
In the era of "Internet +" and Big Data, it is of great practical significance on how to build a training platform that accurately matches the professional development of maker teachers, and to carry out personalized mobile training for maker teachers under the Big Data analysis technology. The construction of the maker teacher mobile training platform, based on the big data analysis technology, is designed to explore the personalized needs of maker teachers in professional development. It introduces a new concept of MOOC and community space design to build the maker mobile training platform framework structure, which contains three layers: application layer, service layer, and data layer. It designs five functional modules: diagnostic demand analysis module, personalized service customization module, online maker course module, seminar space module, and evaluation feedback module. The case analysis of the platform and its application effect shows that the maker teacher mobile training platform based on big data analysis has obvious effects on professional development for teachers and can provide reference for future research on related topics.
\end{abstract}

\section{INTRODUCTION}

As the forerunner in the process of education reform, the improvement of teachers' professional quality is the core to ensure the quality of education. Maker Education, as a "new normal" education, is changing traditional educational concepts, organizations, models and methodologies, leading a wave of educational revolution sweeping the globe. According to the "Investigation on the Status Quo of the Development of Maker Space in Primary and Secondary Schools in China", the subject background of maker instructors in maker education in primary and secondary schools in China is that information technology accounts for $85.51 \%$, general technology $53.62 \%$, physics $33.33 \%$, Mathematics $20.29 \%$, biology $15.94 \%$, and others $23.19 \%$ (Zhong Baichang, 2016). It is not difficult to find out that the main problem in the development of maker education in China is the contradiction between the increasing number of students and the shortage of high-quality maker teachers. Teachers of two disciplines, information technology and general technology, have become the main force of maker education in China's primary and secondary schools at this stage, but it is not enough for the development of maker education if it is only to provide guidance to students from the technical level. In 2010, the "National Training Program for Primary and Secondary School Teachers" was implemented. Through innovative training mechanisms, the combination of off-the-job training, centralized training and large-scale distance training for key teachers has greatly improved the overall quality of primary and secondary school teachers (Liang Wenxin, 2017). Nevertheless, the large-scale distance training is still unsatisfactory in terms of training effects, and there are problems such as neglecting the enthusiasm of trainees, lack of logic in curriculum design, etc. leading to high consumption and low efficiency (Li Yunfu, 2016). Judging from the current teacher training platforms, the analysis of the needs of trainers in the early stage often determines the setting of later training goals and the design and development of training courses and resources. However, the teacher training under the inherent thinking mode often ignores the early stage. The analysis of training needs focuses on the implementation of training programs, which is difficult to adapt to the new normal of maker teacher training in the "Internet +" and Big Data era. Therefore, at this stage, relying on network technology to build a mobile training platform that accurately matches the personalized training needs of primary and secondary maker teachers, and emphasizing the improvement of the quality of maker teachers has become the primary issue to promote the development of the maker education.

\section{THE OPPORTUNITY BROUGHT BY BIG DATA ANALYSIS TECHNOLOGY TO DETERMINE THE PERSONALIZED DEVELOPMENT NEEDS OF MAKER TEACHERS}

\subsection{Exploring the implicit demand of maker teachers in professional development by analyzing teacher behaviour data}

The implicit demand for professional development of maker teachers refers to the fact that maker teachers cannot actively discover and express themselves, and need to use data mining technology for analysis and clarification. At the level of user implicit demand mining and analysis, Big Data analysis technology has a complete chain of actions, especially the analysis of various user behaviour data as a basis to better help learners understand their invisible needs (Sandryhaila, A. 2014). In the field of education, many MOOC platform operators have begun to try to find favourable data for research through the huge information generated during the learning process of learners, and then to obtain the most clicked learning resources for learners and the most repeated video clips of courses as well as learning content with high error rates, the results are analyzed and transmitted to the course development team to help them adjust the course schedule in a timely manner and improve the teaching strategy (Guo, P. J. 2014). This also shows from the side that big data analysis technology can provide more real and reliable demand diagnosis. In addition, $\mathrm{Gu}$ Xiaoqing and others used "micro-video" teaching experiments to capture and quantify the learning process data of learners from multiple dimensions, and characterize the learning behaviour of learners ( $\mathrm{Gu}$ Xiaoqing, 2016); An analysis report

\footnotetext{
* Corresponding author
} 
on the improvement of teachers 'teaching behaviours, and then found hidden needs for teachers' professional growth (Wang Lu, 2017). It is not difficult to find out that the above-mentioned requirements are all determined by the use of technical equipment through the observation and recording of user behaviour, and then by the big data analysis technology to further determine the implicit demands of users.

2.2 Finding the explicit demand of Maker teachers in professional development through data analysis of problems arising in the teaching process

The explicit demand for professional development of maker teachers refers to the needs that can be clearly expressed by maker teachers and can be directly obtained from various types of teaching problems that maker teachers face in the teaching process (Darling-Hammond, L. 2011). Researchers synthesize data from various aspects of feedback from maker teacher groups to conduct big data analysis, and systematically summarize the common problems of teacher groups with a certain attribute. Therefore, based on the problem data provided by the maker teacher's teaching practice, using the advantages of big data technology to analyze and sort it out, we can determine the needs and key issues of the maker teacher group to achieve professional development.

\section{ANALYSIS OF MAKER TEACHER GROUP TRAINING NEEDS: THE STARTING POINT OF MOBILE TRAINING PLATFORM CONSTRUCTION}

In order to understand the training needs of the maker teacher group in primary and secondary schools, the research team adopted a survey method combining interviews and questionnaires in June 2017, which were designed for the investigation on four aspects: the basic conditions of teachers, training resource requirements, training service requirements and training platform function requirements. The study selects the Cronbach's alpha coefficient as the reliability parameter, and calculates the information parameter as 0.885 , which is greater than 0.8 , indicating that the questionnaire has high reliability. The principal factor analysis method is used to obtain the common factor variance of each item is greater than 0.7 by extracting 12 public factors, the cumulative variance explanation amount reached $69.53 \%$, and the general education measurement statistics reached more than $60 \%$, which is good, indicating that the questionnaire is well-structured and has good validity.

The information technology teachers who participated in the survey accounted for $62.46 \%$, general technology and comprehensive practice teachers accounted for $12 \%$ and $5.65 \%$ respectively, and most of them were teachers engaged in STEM education such as physics, science, and mathematics. A survey of existing learning foundations found that $32.31 \%$ of primary and secondary school teachers stated that they had studied relevant courses and technologies and had established makerrelated courses, and $30.69 \%$ of teachers had some knowledge of maker education, only $14.51 \%$ of teachers said they have never learned any maker technology or courses. In the survey of training resource requirements, it was found that the needs of maker teachers in primary and secondary schools for interpretation of the maker concept, explanation of maker project production cases, maker course design and production, and maker space construction ideas are high; Arduino open source hardware and 3D printing technology and Scratch programming learning 3 types of learning carriers have higher learning needs; $75.04 \%$ of teachers hope to support micro-video format at the level of learning materials support, $95.56 \%$ of teachers want to provide some maker software resource packages as well as learning website links. In the training service demand survey, it was found that $78 \%$ of teachers hope to regularly obtain resources, services, and activities pushed by the platform through a combination of online, offline, and multi-channel methods. They also hope to use new media platforms such as WeChat and Weibo to transfer the platform's new resources and new activities are pushed to students in a timely manner; $14 \%$ of teachers hope that the training team can enter the school, regularly provide academic lecture services, and accurately locate the personalized needs of teachers. A survey of the functional requirements of the training platform found that $52 \%$ of the course learning function requirements, $67 \%$ of real-time interactive functions, $15 \%$ of resource sharing functions, and $26 \%$ of others. In summary, nearly half of the teachers have a certain understanding of maker education and have learned related technologies and a certain learning foundation. They should have a certain gradient in the difficulty of designing teacher training courses, and include an introduction to maker education. At the same time, it should also include high-level learning resources; at the same time, in the setting of training content, we must pay attention to the combination of teaching practice and educational theory, select universal learning carriers, and build links with learning websites, learning software resource packs, video courses and diversified training support service platform included as well..

\section{ANALYSIS OF MAKER TEACHER GROUP TRAINING NEEDS: THE STARTING POINT OF MOBILE TRAINING PLATFORM CONSTRUCTION}

At present, the rapid growth of data and the innovation of thinking methods have provided favourable conditions for the reform of teacher training. The gradual maturity of big data analysis technology provides technical supports to teachers who deeply explore the teaching process, spot teaching problems, and accurately match different learning needs. Therefore, this study is guided by big data thinking for personalizing mobile training platform for primary and secondary maker teachers and is divided into three dimensions: Application Layer, Service Layer and Data Layer. The core system in Figure 1 shows that the platform focuses on the relationship between user demand collection, training programs, and functional services.

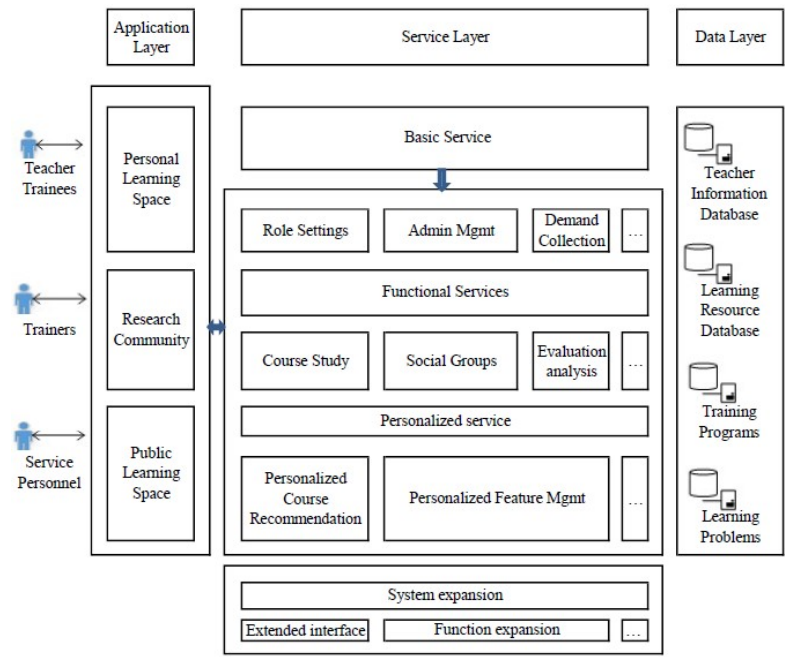

Figure 1. The Overall Framework of the Maker Teacher Mobile Training Platform 


\subsection{Application layer}

The main function of the application layer is to provide different access points for various user groups and provide various training support. Among them, the service personnel are mainly responsible for the operation and maintenance of the training platform, and dynamic management and setting of the information of the platform users; the training teachers are mainly responsible for supervising the learning process of the participating teachers, and feedback on the quality of the learning, etc. Teachers participated should carry out self-study in their own personal cyberspace and participate in the communication and collaboration between the training community and public space (Zeng Mingxing, 2017). This layer has two characteristics of supporting multi-terminal access and supporting personalized learning. In order to make the learning activities of the participating teachers not restricted by time and space, the platform supports the access of multiple terminals such as PC and mobile terminals. At the same time, trainee teachers can manage their own personalized resources such as training programs and courses through personal online learning spaces and online training communities, and can also communicate with other trainee teachers through public online learning spaces to share learning resources and experiences to achieve personalized learning.

\subsection{Service layer}

As the core part of the training platform, the service layer is the key path to connect users and resources. The service layer mainly provides 4 types of services: basic services, functional services, personalized services, and system expansion services. The basic services mainly include demand information collection, role management, and permission settings, etc. The purpose is to provide service personnel with the management of user access to platform functions and resources. The main purpose of functional services is to provide training-related services for participating teachers, including course learning, social groups, evaluation analysis, etc. The personalized service provides the following information service functions: course recommendation service, data literacy training, and tracking service guidance. System expansion services include two types: server interfaces and functional interfaces. The main purpose is to facilitate the expansion and integration of the training service platform and provide functional interfaces to the platform.

\subsection{Data layer}

The data layer, as the foundation layer of the platform, integrates the teacher information database, training program set, learning resource library, etc., and is the physical layer that stores platform-related data. The teacher information database mainly stores the basic information and learning needs of the participating teachers. The learning resource library mainly stores various multimedia learning resources designed and developed for participating teachers. The training program set analyzes the classification of the maker teacher group and the aggregation of training needs information at the application layer, clarifies the basic information of different types of maker teacher groups, the training contents, and specific practical project settings.

\section{DIVERSIFIED INTEGRATION: THE CORE FUNCTION DESIGN OF PERSONALIZED MOBILE TRAINING PLATFORM}

Based on the above analysis of the personalized training needs of primary and secondary maker teachers, the researchers divided the functional modules of the personalized mobile training platform for primary and secondary maker teachers into diagnostic demand analysis modules, personalized service customization modules, online maker course modules, research space module, and evaluation feedback module.

\subsection{Diagnostic requirements analysis module}

The analysis of the training needs of the participating teachers is the core function of the personalized training mobile platform. Its main task is to objectively recognize the participating teachers and classify the participating teacher groups in order to carry out targeted personalized training. This module tests personal characteristics such as the knowledge structure and ability level of the participating teachers. It mainly tests the general knowledge and software operation knowledge used in the maker learning process, and initially determines the different types of maker teachers. It also determines what types of training contents should be applied to the participating teachers so as to provide a reference for customized training programs and course recommendations.

The module's analysis of training demand data is mainly carried out in two aspects: on the one hand, the use of related information mining and analysis technology on teacher personal data to determine the explicit training needs under short-term and long-term goals; The behaviour data is deeply mined, and the teacher's own status and information situation are obtained according to the data analysis process of data miningassociation-statistics-analysis, and then the hidden information needs of teachers are determined. Teachers' training needs are highly related to their teaching goals, but during the teaching process, users are prone to produce temporary information needs due to the external environment. This requirement may deviate from or even conflict with their goals. Based on this, this paper proposes Framework of information demand model for teacher group (Dong Tongqiang, 2017) (see Figure 2). Teachers' information needs are composed of explicit needs and implicit needs. The implicit needs are mainly formed by the teacher's information behaviour data and resource management data mining. After the information needs are determined, they are integrated into the information needs vector and are related to the teacher's real-time needs. Establish a feedback update mechanism (Kovalchick, A. 2004).

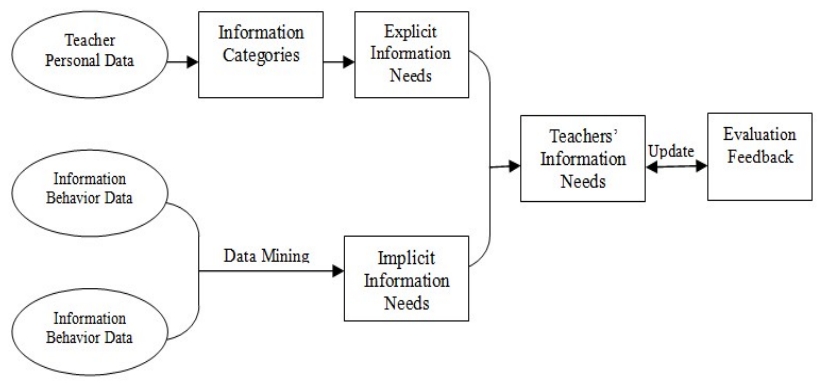

Figure 2: Framework of Information Demand Model for Maker Teachers 


\subsection{Personalized service customization module}

The personalized customized service module is based on the diagnostic needs analysis module and the screened scientific data; it can accurately personalize service if needed. Artificial intelligence technology with neural network algorithms is to analyze user needs information from the user's interest information base, inferring user intent and labelling the types of preferences. Based on this, it can classify the user group and identify the dynamic demands of different users through smart tags. By data prediction and analysis methods, it can analyze user behaviour trends; visualize the presentation from user behaviour data to user behaviour characteristic information. Under the intelligent push service, it also determines personalized service products for different user groups and efficiently pushes users training information resources, as well as to adjust training service supply contents through evaluation feedback mechanism.

\subsection{Online maker course module}

The online maker course modules mainly include online maker teaching modules and online maker learning modules. With the integration of main courses, teacher trainees, personalized service and the maker resource construction, it creates a networked, personalized, and intelligent cloud learning environment for the maker community (Zhu Pengwei, 2018). Its main functions include resource navigation, course browsing, course textbook uploading, crowd-creation space design, onestop retrieval, etc. By integrating learning resources inside and outside the school, it can give full play to the role of the platform in the daily teaching and training of maker teachers. In this module, we mainly rely on the WeChat public platform to support the sharing and learning of course resources. We design and upload micro-video course resources through the background management system, such as Arduino open source hardware and 3D printing technology, Scratch programming learning and other creative courses. Maker teachers can search all the resources on the platform through the search interface, find the corresponding course resources by selecting the corresponding course tags, and browse the courses. After selecting the course resources of interest, they may browse relevant information of the course to decide whether to continue to learn the course. The production of the curriculum is based on the requirements of the MOOC curriculum, and micromaterial training resources such as micro-projects, micro-cases, and micro-experiments are added to assist their learning. The system implements differentiated teaching through real-time recording, statistics and analysis of the learning situation of its login to the micro-site, and automatic scoring.

\subsection{Discussing module}

Situational cognition and learning theory emphasizes the importance of creating functional situations for learners (Cobb, P.1999). Maker teachers must carry out teaching and research activities into specific situations. And the problem of how to build real-time interactive peer collaboration situations for them is the beginning of platform design. In this study, the researchers divided multiple learning groups according to the pre-test results of the teachers participating in the training through the platform to meet the real-time interaction and collaborative learning among the teacher groups, and established a real-time collaborative interaction mechanism in group members. The functions of online chat, WeChat group, and circle of friends of social software realize the interactive community among teacher groups, forming understanding of knowledge in the process of interactive activities, collaborative learning and competitive learning in a virtual community environment. That helps teachers form a learning community with common interests, and realize the generation and creation of knowledge. At the same time, through the form of building a training community, participating teachers are encouraged to upload their own research reports, research papers, experiences, etc., which realizes the co-construction and sharing of learning resources.

\subsection{Evaluation Feedback Module}

After obtaining the learning resources and service information provided by the platform, the participating teachers can use the platform's evaluation function to comment on whether the learning resources are accurately pushed and whether they meet their learning needs, and they can also submit their own opinions and suggestions in the message area (Yang Sen, 2015). These data have provided a basis for the resource aggregation of the later platform and the optimization of information pushes. In addition, you can also make suggestions to the developers of the platform through feedback, and participate in the development of the platform service products or tools.

\section{CASE STUDY}

\subsection{Implementation of functional modules}

Maker Teacher WeChat Academy is a service platform for researchers' mobile training built on the WeChat public platform by researchers, which includes three first-level menu modules: "Learn • Maker", "Do • Maker" and "View • Maker", each of which contains second-level menu modules such as micro-curriculum, creative training, maker, etc. Among them, the five main function modules that are more commonly used are as follows:

Smart Reply: When the user enters the keywords of the subject resource content in the platform which will automatically push the content of such resources.

Resources Crowdfunding WeChat Wall: Using fashionable barrage technology, developers will publish course resource titles and training outlines to many learners in the early stage of platform construction, and judge whether it is necessary to open them based on the feedback of learners. At the same time, learners, based their interests, can also s provide developers with a list of what they need to learn.

Evaluation and Investigation: It is used to evaluate the works of participating teachers and self-evaluation or mutual evaluation among groups. It can also be used for questionnaires to ask the participating teachers' needs for platform push resources and suggestions for platform construction. All the answers are with detailed statistics in the background platform.

Micro Courses: It is mainly used by the participating teachers to learn maker education theory, projects, and cases, covering Scratch creative programming, creative robots, 3D creative printing and other contents.

Microsite: It is mainly used for participating teachers to learn maker courses online and to record statistics and analyze their learning situation on the micro website. 
These functions and operations can be completed quickly by WeChat public account. With these functions, the trainers can manage the mobile learning situation and project design of the participating teachers in real time, and can also achieve a good interaction among the teachers.

\subsection{Analysis of the application of maker teacher WeChat}

Since July 2017, this platform has been applied to the "Research on the Application of Maker Education and 3D Printing Education" for key maker teachers in primary and secondary schools in the $\mathrm{R}$ region, and it aims to enhance teachers' innovation learning for course designing with creative skills. Relying on the WeChat learning platform of the Maker Academy, the three menu modules of "Learn • Maker", "Do • Maker" and "View - Maker" divide the course content into 3 parts: (1) Relevant theories in the field of Maker Education Exploration, (2) 3D printing-related knowledge and skills guidance and (3) 3D printing industry development, education applications and entrepreneurship-related knowledge. Based on this, the researchers briefly introduced the application of the training platform from three aspects as: Pre-Training, During Training, and Post Training.

\subsubsection{Pre-Training: preview feedback and adjust teaching}

Before the training, the training teachers will pre-design the course content in advance and use the form of a combination of "video + text + pictures" multiple media to post real-time notifications to trainee teachers through the WeChat platform 3 days before the start of the course. Teachers are trained to join different study groups according to their own learning interests. At the same time, a class learning communication WeChat group is set up, which provides a personalized learning space for them and also provides a group wisdom environment for the teacher group. After completing the pre-reading task within the prescribed time, and participating in the feedback to the platform, the tracking and recording function of the platform are analyzed for the teacher's learning behaviour, and it later provides training teachers with feedback analysis of each participating teacher's learning situation.

\subsubsection{During Training: Online and offline interactive teaching, exploration and exchange}

In the classroom, the training teacher uses the mobile learning function circle composed of multiple functional modules such as WeChat Wall, WeChat community, WeChat website, keyword response, group function, etc., and divides the training class into three parts: (1) Basic knowledge. The training teachers combined with the feedback from the participating teachers on the preview content, and directly expressed the maker education theoretical knowledge and 3D printing-related theoretical knowledge to the learners in the form of live lessons. (2) Class interaction. Before the lesson, the training teacher has added video courses and other materials to the platform's material library and voting module in the form of materials, and conducted classroom interaction with the participating teachers in the form of keyword responses. For example, "Teachers use the working principle of 3D printers" as classroom inquiry tasks, organize participating teachers to participate in the course learning and vote; after the voting is completed, relevant data will be uploaded to the platform, and they will jointly analyze the voting results so as to explore and form collective wisdom. At the same time, the training teacher can use the WeChat Wall to provide a discourse channel for the participating teachers, the participating teachers can send questions, and after the training teachers have reviewed it, they can "go to the wall", and other teachers can reply and discuss the problems on the WeChat Wall to achieve real-time interactive feedback. (3) Autonomous collaborative learning. Participating teachers use self-organized learning activities organized by the interest groups and microwebsites and micro-communities on the previous platform to interact with their learning partners to discuss their confusion in the training classroom and establish a collaborative learning community; Participating teachers can also post in the Q \& A discussion forum in the WeChat community. All teachers can obtain the information posted by them and express their opinions in the form of follow-up posts. If the help of experts is needed, they only need to use the "@ expert" to ask the experts who will give feedback in time after obtaining the information. Participating teachers can also use the platform to construct a learning method for the social group discussions, so that they can obtain creative inspiration in interactive feedback, and instead create new works independently.

\subsubsection{Post Training: creative sharing, continuous evaluation}

In order to cultivate the higher-order thinking ability of trainee teachers, trainers can push specific maker projects to trainee teachers in the form of tasks through the platform's automatic push function after school. Teachers choose corresponding projects based on their own learning situation and interests to form a community of practice. The members of the community start learning and manufacturing according to the four-link iterative loop model of "research and analysis, formulating plans, prototype design, communication and reflection" (Zhen Yanlin, 2011), and assign learning tasks according to the interests of each member in the community. In the practice of the project, the participating teachers can communicate with the members of the community in real-time in a voiced narrative manner to solve each other's problems, and the trainers can obtain the project's production process at any time through the platform's real-time tracking and recording function. When problems arising, and then trainers can give the project community of practice specific guidance and process evaluation. After the project is completed, the platform will display the results by the voting function of the platform and the microcommunity, thus, the evaluation will be conducted through a combination of community self-evaluation, mutual evaluation and expert evaluation.

\subsection{Application effect analysis of platform}

In order to further understand the platform's application effect and training effect feedback, the researchers conducted interviews and questionnaire surveys on 67 primary and secondary maker teachers participating in the training from two aspects of the platform's use effect satisfaction and teaching effect. Through three aspects of teaching activities, objectives and content, a questionnaire is applied to analyze users 'satisfaction with the operation of the personalized mobile training platform, interactive communication satisfaction, and overall satisfaction with the platform. At the same time, through interviews, from the teachers' communication ability and group collaboration ability, hands-on ability, innovation ability and other dimensions were used to investigate the teaching effect. The research used SPSS 22.0 to analyze the reliability and validity of the questionnaire. The statistical analysis results show that $92.56 \%$ of the students are satisfied with the operation of the mobile training platform, $91.45 \%$ are satisfied 
with the degree of interaction and communication, and $89.70 \%$ are satisfied with the use of the personalized mobile training platform. This fully shows that compared to the traditional offline training method, most teachers prefer to rely on WeChat public platform for mobile training, but $10.30 \%$ of the participating teachers are still not very satisfied with the use of the platform. Through communication and understanding, some teachers said that the reason why they chose to use WeChat platform for mobile training is that when they encountered difficulties or entered a misunderstanding in the maker course offline, experts and learning partners could help them solve the problem through WeChat platform in time and provide guidance. The researchers also found some shortcomings in the application of the platform in the interviews: (1) the topic discussions were lack of the supervision by trainers in the platform group. The related investigations show that if the trainers can control the group topic discussions in a timely and reasonable manner during the training process, it can prevent the discussion deviates from the topic and becomes a "chat place" for learners, which will greatly promote learning discussions and create a better learning environment for learners. (2) The training of teachers' independent innovation ability and practical ability needs to be strengthened. Researchers have found that individual teachers have opportunistic and inactive participation in group activities during the learning process. In the process of project practice, they are often lazy to think while just depending entirely on the help of learning partners and experts in the community. In this regard, in the next teaching activities, the corresponding participating teachers should be warned, the peer evaluation mechanism and mutual supervision among learning peers should be strengthened.

\section{CONCLUSION}

The lack of maker education teachers has become a "short board" that limits the development of maker education, but the training of maker teachers is not a project that can be completed overnight. From a macro perspective, the professional training of maker teachers is more like a systematic project, which requires unified planning and design at the national level, as well as the support of specialized mechanisms, universities, and training institutions. From a micro perspective, at this stage, information technology teachers and general technology teachers are still the main groups engaged in maker education in schools. Selecting some of them for short-term online and offline short-term training is an expedience to solve the current shortage of teachers. This research is guided by problemoriented and big data thinking, and builds a personalized mobile training platform for maker teachers in the "Internet +" and Big Data era, which provides maker teachers with a full range of personalized services for demand prediction and adapt to multiple changes. However, at present, China's artificial intelligence technology and maker teacher training services are still in the exploratory and initial stages. So, it is a right time to framework how to realize the seamless integration of intelligence and maker teacher training services in a big data era, which requires further consideration in the practice process.

\section{REFERENCES}

Zhong Baichang., 2016. Talking about the background, essence, form and support system of maker education $[\mathrm{J}]$. Modern Educational Technology, 2016, (6): 13-19.
Liang Wenxin., 2017. Design of Mobile Training Courses for Teachers Based on Big Data [J]. China Educational Technology, 2017, (06): 120-123.

Sandryhaila, A., \& Moura, J. M., 2014. Big data analysis with signal processing on graphs. IEEE Signal Processing Magazine, 31(5), 80-90.

Guo, P. J., Kim, J., \& Rubin, R. (2014, March). How video production affects student engagement: An empirical study of MOOC videos. In Proceedings of the first ACM conference on Learning@scale conference (pp. 41-50). ACM.

Li Yunfu, Yang Xiaohong. Research on O2O Teacher Training Model Based on Big Data Analysis: Preliminary Thinking of "Internet + " Teacher Training [J]. China Educational Technology, 2016, (12): 113-120.

Gu Xiaoqing, Shu Hang., 2016. Has the role of information technology happened-characteristics of learning behavior with learning analysis technology $[\mathrm{J}]$. Remote Modern Education Research, 2016, (05): 10-19.

Wang Lu, Li Yao., 2017. An analysis of teaching phenomena under the perspective of big data in classroom teaching behaviors [J]. Research on Electronic Education, 2017, 38 (04): 77-85.

Darling-Hammond, L., Amrein-Beardsley, A., Haertel, E. H., \& Rothstein, J., 2011. Getting Teacher Evaluation Right: A Background Paper for Policy Makers. National Academy of Education (NJ1).

Zeng Mingxing, et al., 2016. Construction of online learning space for college student makers [J]. China Educational Technology, 2016 (11): 30-38.

Dong Tongqiang, Ma Xiufeng, Zhang Yongpeng., 2017. Design of Intelligent Embedded Service Platform for University Library for Maker Groups [J]. Researches in Library Science, 2017, (15): 83-88.

Kovalchick, A., \& Dawson, K. (Eds.)., 2004. Education and Technology: AI (Vol. 1). Abc-clio.

Zhu Pengwei., 2018. Research on the Construction of University Discipline Service Platform from the Perspective of "Internet + Library" [J]. Information Science, 2018, 36 (04): 91-94 + 110 .

Cobb, P., \& Bowers, J., 1999. Cognitive and situated learning perspectives in theory and practice. Educational researcher, 28(2), 4-15

Yang Sen., 2015. Design and implementation of a resource platform for personalized teaching [D]. Huazhong Normal University, 2015.

Zheng Yanlin, Li Luyi., 2011. Construction and practice of "integrated" information technology teacher training model in primary and middle schools [J]. China Educational Technology, 2011 (11): 58-61. 